\title{
Error-Dependent Data Scheduling in Resource-Aware Multi-Loop Networked Control Systems *
}

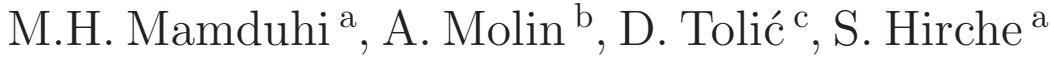 \\ ${ }^{a}$ Chair of Information-oriented Control, Technical University of Munich, Arcisstraße 21, D-80290 München, Germany \\ ${ }^{\mathrm{b}}$ ACCESS Linnaeus Centre, KTH Royal Institute of Technology, SE-100 44 Stockholm, Sweden \\ ${ }^{\mathrm{c}}$ Department of Electrical and Computer Engineering, The University of Dubrovnik, Cira Carića 4, 20000 Dubrovnik, Croatia
}

\begin{abstract}
In this work we address the problem of event-based data scheduling for multiple heterogeneous LTI control loops over a shared resource-constrained communication network. We introduce a novel bi-character scheduling scheme, which dynamically prioritizes the channel access at each time-step according to an error-dependent priority measure. Given local error thresholds for each control loop, the scheduling policy deterministically blocks the transmission from sub-systems with lower error values. The scheduler then allocates the limited communication resource probabilistically among the eligible sub-systems based on a prioritized measure. We prove stochastic stability of the networked control system under the proposed scheduler in terms of $f$ ergodicity of the overall network-induced error. Uniform analytical performance bounds are further derived for an average cost function comprised of a quadratic error term and transmission penalty. The simulation results show that our approach results in a significant reduction of the aggregate network-induced error variance compared to the conventional scheduling protocols.
\end{abstract}

Key words: Networked control systems, Markov chain; error state; state-dependent scheduling; shared communication resource.

\section{Introduction}

Traditional digital control systems are typically associated with time-triggered control schemes and periodic sampling. The introduction of communication networks for data transmission between distributed entities in large-scale systems spurs the design of more advanced sampling strategies that result in more efficient utilization of resources. However, control over shared communication resources imposes several design challenges due to bandwidth limitations, congestion, collisions, delays and dropouts [9]. Many recent results $[1,6]$ suggest that it is often more beneficial to sample upon the occurrence of specific events, rather than after a fixed period of time elapses, especially when dealing with scarce resources. The design of scheduling rules with periodic and aperiodic information updates is an active field of re-

\footnotetext{
* The material in this brief was partially presented at 21 st International Symposium on Mathematical Theory of Networks and Systems, 2014. Corresponding author M.H. Mamduhi. Tel. +4989-289-25738. Fax +4989-289-25724.

Email addresses: mh.mamduhi@tum.de (M.H. Mamduhi), adammol@kth.se (A. Molin), domagoj.tolic@fer.hr (D. Tolić), hirche@tum.de (S. Hirche).
}

search $[11,12,16,17,22-24]$. It is shown that eventtriggered schemes often outperform time-triggered laws in terms of resource consumption while preserving the same level of control performance $[11,16,22,24]$. The efficiency of the event-based approaches in multi-loop NCSs, where multiple sub-systems compete for the communication resource, is even more evident $[3,12,16,17]$. Try-Once-Discard (TOD) is a basic deterministic eventbased scheduling law which awards the channel access to the system with the largest estimation error and discards the remaining transmission requests [23]. Stability criteria for such systems are based on the Maximal Allowable Transfer Intervals (MATI) [18,23]. Approaches investigating stochastic stability of NCSs under eventbased rules are presented in $[7,12,21]$. Deterministic scheduling policies usually render improved performance in comparison with randomized ones as they award the channel to systems with the highest priority. However, they often lack scalability and flexibility in dealing with channel imperfections and might not be convenient for practical realizations [4]. The majority of works, with notable exceptions in [2, 3, 12, 16, 19], consider event-based scheduling policies for single-loop NCSs. Results on stability of multi-loop NCSs with event-based scheduling rules are found in $[12,16,19]$. 
Scheduling mechanisms can be realized in a centralized or distributed fashion. Time division multiple access (TDMA), and code division multiple access (CDMA) are two common centralized protocols often preferred in small and medium-size networks. They offer collision-free and precise channel scheduling with higher throughput, while they consume less energy compared to e.g. CSMA-CA policy, where each node senses the channel permanently [20]. Furthermore, bandwidth arbitration is facilitated as they can prioritize channel access. However, they lack flexibility and scalability and are not suitable for large-scale networks due to their synchronous nature. Distributed approaches, represent easy-to-install, low-cost and scalable scheduling design suitable for NCSs with a large number of loops. However, collisions take place inevitably within distributed protocols and need to be handled with care in the NCS design. To exploit the advantages of both protocol types, hybrid designs are becoming evermore popular $[8,20,25]$. We introduce a novel event-based bi-character scheduling rule for NCSs composed of multiple stochastic LTI control loops sharing a common communication medium. The proposed scheduler promises more efficient use of the scarce resource in comparison with conventional schemes. In our design, the scheduler deterministically precludes transmission requests of sub-systems with errors not exceeding pre-specified local thresholds. Afterwards, the channel is allocated probabilistically among sub-systems qualified for transmission, according to an online error-dependent priority measure. Since the local errors are driven by the Gaussian noise, transmissions occur randomly under an event-based rule. Consequently, by deterministically blocking the sub-systems with smaller local errors, the performance enhancement is attained. We show stochastic stability of the multiloop NCSs in terms of $f$-ergodicity of the underlying error Markov chain. In addition, we derive analytical upper bounds for an average quadratic cost function. In the remainder, Section 2 presents the problem of interest and provides necessary preliminaries. In Section 3, stability of NCSs under the proposed policy is studied. Performance analysis is then presented in Section 4. Finally, numerical results are illustrated in Section 5.

\section{Problem Statement and Preliminaries}

Consider a set of $N$ heterogeneous LTI control loops coupled through a shared communication channel as depicted in Fig. 1. Each individual loop consists of a discrete time linear stochastic sub-system $\mathcal{P}_{i}$ and a controller $\mathcal{C}_{i}$, where the link from $\mathcal{P}_{i}$ to $\mathcal{C}_{i}$ is closed through the shared communication channel. A scheduling unit decides when a state vector $x_{k}^{i} \in \mathbb{R}^{n_{i}}$ at time-step $k$ is to be scheduled for channel utilization, where $n_{i}$ is the dimension of the $i^{\text {th }}$ sub-system. The LTI plant $\mathcal{P}_{i}$ is modeled by the following stochastic difference equation:

$$
x_{k+1}^{i}=A_{i} x_{k}^{i}+B_{i} u_{k}^{i}+w_{k}^{i},
$$

where $w_{k}^{i} \sim \mathcal{N}(0, I)$ is i.i.d. at each time $k$, and for each sub-system $i$, while constant matrices $A_{i} \in \mathbb{R}^{n_{i} \times n_{i}}$ and $B_{i} \in \mathbb{R}^{n_{i} \times m_{i}}$ describe system and input matrices of sub-system $i$, respectively. Initial state $x_{0}^{i}$ is randomly chosen from an arbitrary bounded-variance distribution. The overall network initial state $x_{0}$, together with the overall noise sequence $w_{k}$, generates a probability space $(\Omega, \mathcal{A}, \mathrm{P})$, where $\Omega$ is the set of all possible outcomes, $\mathcal{A}$ is a $\sigma$-algebra of events associated with probability $\mathrm{P}$. The variable $\delta_{k}^{i} \in\{0,1\}$ represents the scheduler's decision on whether a sub-system $i$ transmits at a time-step $k$ :

$$
\delta_{k}^{i}= \begin{cases}1, & x_{k}^{i} \text { is sent through the channel } \\ 0, & x_{k}^{i} \text { is blocked. }\end{cases}
$$

We assume a loss-less channel, i.e. if a packet is transmitted, it will not be dropped. Data scheduling over lossy channels is investigated in [14]. It is assumed that the $i^{\text {th }}$ controller merely has local knowledge of $A_{i}, B_{i}$, and the distributions of process noise $w_{k}^{i}$ and $x_{0}^{i}$, where the pair $\left(A_{i}, B_{i}\right)$ is stabilizable. The control law $\gamma^{i}$ is described by a measurable and causal mapping of past observations:

$$
u_{k}^{i}=\gamma_{k}^{i}\left(Z_{k}^{i}\right)=-L_{i} \mathrm{E}\left[x_{k}^{i} \mid Z_{k}^{i}\right],
$$

where $Z_{k}^{i}=\left\{x_{0}^{i}, \delta_{0}^{i}, \ldots, x_{k}^{i}, \delta_{k}^{i}\right\}$ is the $i^{\text {th }}$ controller observation history, and $L_{i}$ is the feedback gain. A modelbased estimator computes the state estimate if $\delta_{k}^{i}=0$ :

$$
\mathrm{E}\left[x_{k}^{i} \mid Z_{k}^{i}\right]=\left(A_{i}-B_{i} L_{i}\right) \mathrm{E}\left[x_{k-1}^{i} \mid Z_{k-1}^{i}\right],
$$

with $\mathrm{E}\left[x_{0}^{i} \mid Z_{0}^{i}\right]=0$. The network-induced error $e_{k}^{i} \in \mathbb{R}^{n_{i}}$ is defined as $e_{k}^{i} \triangleq x_{k}^{i}-\mathrm{E}\left[x_{k}^{i} \mid Z_{k}^{i}\right]$. Employing (1)-(3), and the definition of the estimation error $e_{k}^{i}$, results in

$$
\begin{aligned}
x_{k+1}^{i} & =\left(A_{i}-B_{i} L_{i}\right) x_{k}^{i}+\left(1-\delta_{k}^{i}\right) B_{i} L_{i} e_{k}^{i}+w_{k}^{i}, \\
e_{k+1}^{i} & =\left(1-\delta_{k+1}^{i}\right) A_{i} e_{k}^{i}+w_{k}^{i} .
\end{aligned}
$$

It follows from (4) that if the $i^{\text {th }}$-loop is closed at time $k$, i.e. $\delta_{k}^{i}=1$, the stabilizing gain $L_{i}$ ensures the closedloop matrix $\left(A_{i}-B_{i} L_{i}\right)$ is Hurwitz. Moreover, (5) indicates that the evolution of $e_{k}^{i}$ is independent of the system state $x_{k}^{i}$ and control input $u_{k}^{i}$. Define $\left[x_{k}^{i \top} e_{k}^{i \top}\right]^{\top}$ as the aggregate state of sub-system $i$. Stability of a closedloop system $i$, however, does not imply convergence of the error state $e_{k}^{i}$. Hence, given a stable closed-loop matrix $\left(A_{i}-B_{i} L_{i}\right)$, showing convergence of $e_{k}^{i}$ suffices to show stability of sub-system $i$ with the aggregate state $\left[x_{k}^{i \top} e_{k}^{i \top}\right]^{\top}$. We show later that if $A_{i}$ is unstable, then the $i^{\text {th }}$ loop needs to be closed "often enough" over an interval to ensure a converging error dynamics. This separation enables us to design the scheduler, which affects the error state $e_{k}^{i}$, independently from the control law $u_{k}^{i}$. To that end, we employ an emulation-based control strategy with the minimum required assumptions, i.e. stabilizing and linear control law, to ensure the closed-loop systems are stable in the absence of capacity constraint. 
In the scheduling design, the goal is to develop a new scheme which allocates the limited communication resources more efficiently resulting in an improved overall performance, while preserving stability of the stochastic NCS introduced in (1)-(5). We assume that the communication channel is subject to the capacity constraint such that not all sub-systems can simultaneously transmit. Consequently, some of ready-to-transmit data packets are blocked. Here we introduce a novel errordependent scheduling rule that dynamically prioritizes the channel access among the control loops competing for transmission. The scheduler decides on the priorities at each time-step according to all sub-system's latest error states. The following scheduling rule defines the probability of channel access for a sub-system $i$, at a time-step $k+1$, based on the received error values $e_{k}$ :

$\mathrm{P}\left[\delta_{k+1}^{i}=1 \mid e_{k}^{j}, \lambda_{i}\right]= \begin{cases}0 & \left\|e_{k}^{i}\right\|_{Q^{i}}^{2} \leq \lambda_{i} \\ 1 & \left\|e_{k}^{i}\right\|_{Q^{i}}^{2}>\lambda_{i} \wedge j_{\lambda} \leq c \\ \frac{\left\|e_{k}^{i}\right\|_{Q^{i}}^{2}}{\sum_{j_{\lambda}}\left\|e_{k}^{j}\right\|_{Q^{j}}^{2}} & \left\|e_{k}^{i}\right\|_{Q^{i}}^{2}>\lambda_{i} \wedge j_{\lambda}>c,\end{cases}$

where $c<N, \lambda_{i} \in \mathbb{R}_{\geq 0}$, and $j_{\lambda} \in \mathbb{W}$ denote the channel capacity, error threshold, and the number of loops satisfying $\left\|e_{k}^{i}\right\|_{Q^{i}}^{2}>\lambda_{i}$, respectively, and $\|\cdot\|_{Q^{i}}$ represents the weighted norm. The proposed rule possesses a probabilistic-deterministic nature. First, if $\left\|e_{k}^{i}\right\|_{Q^{i}}^{2} \leq \lambda_{i}$ at a time $k$, then no transmission request associated with sub-system $i$ is submitted for time-step $k+1$. This feature helps to allocate the channel more efficiently by excluding the sub-systems for which a transmission is not crucial. If $j_{\lambda} \leq c$, then all eligible sub-systems transmit. Otherwise, the channel is allocated probabilistically until the capacity is reached, and other transmission requests are blocked. Indeed, if $j_{\lambda}>c$, each eligible sub-system associated with its assigned probability takes part in a biased randomization. As an example, consider an NCS with $N=4$, where only two are eligible for transmission at a certain time, while $c=1$. Let the priorities be assigned as 0.8 for sub-system 1 , and 0.2 for sub-system 2 . The biased randomization is then a "single" toss of an unfair coin where head (transmission for sub-system 1), and tail (transmission for sub-system 2) turn up with probabilities $80 \%$ and $20 \%$, respectively. As a result, it is not guaranteed that the sub-system with higher priority transmits, though it is likelier. Furthermore, (6) is a collision-free policy as transmissions are centrally scheduled. For the sake of brevity, assume $c=1$. Therefore:

$$
\sum_{i=1}^{N} \delta_{k}^{i}=1, \quad \forall k \geq 0
$$

The results provided below can easily be extended towards the general case $\sum_{i=1}^{N} \delta_{k}^{i}=c$, where $c>1$. We define the aggregate error state $e_{k} \in \mathbb{R}^{n}$ by stacking the error vectors from all control loops in one vector, as follows:

$$
e_{k}=\left[e_{k}^{1 \top}, \ldots, e_{k}^{N \mathrm{~T}}\right]^{\top},
$$

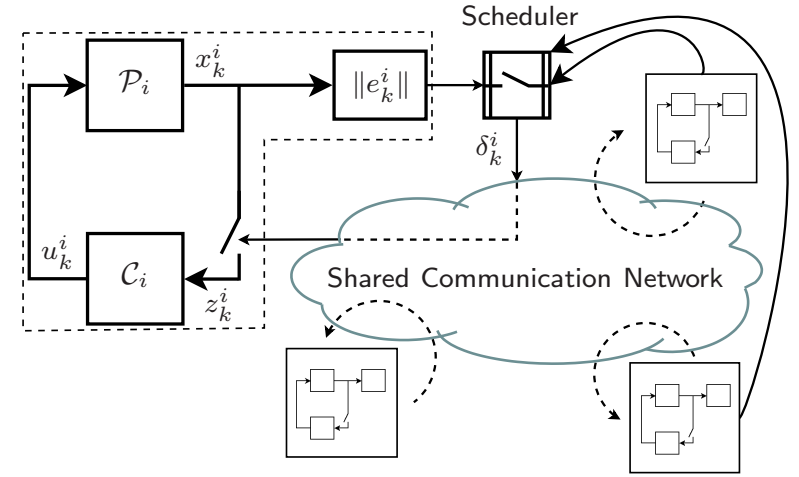

Fig. 1. Multi-loop NCS with shared communication channel.

where $n=\sum_{i=1}^{N} n_{i}$. The scheduling law (6), which generates the input signal for the error state $e_{k}$ according to (5), is a randomized policy depending only on the most recent error values, i.e. the decision on which sub-system eventually transmits at an arbitrary time-step $k+1$ is correlated with the latest error state $e_{k}$. Moreover, the Gaussian noise $w_{k}^{i}$ in (5) has a continuous everywherepositive density function at any element $e_{k}^{i}$ of the overall state $e_{k}$ meaning that there is a non-zero probability to reach any subset of $\mathbb{R}^{n}$. This implies that there exists a transition probability for any event $\mathcal{E} \in \mathcal{A}$ such that $\mathrm{P}\left(e_{k+t} \in \mathcal{E} \mid e_{m}, m<k, e_{k}\right)=P^{t}\left(e_{k+t} \in \mathcal{E} \mid e_{k}\right)$, where $P^{t}\left(e_{k+t} \in \mathcal{E}\right)$ denotes the probability that $e_{k}$ enters a set $\mathcal{E}$ after $t$ transitions, and $m$ is an arbitrary time index before time-step $k$. Since the scheduling policy (6) is forgetful about the error states $e_{m}, m<k$, when deciding the possibility of next transmission via $\delta_{k+1}^{i}$, the stochastic process (8) is a Markov chain. The process is homogeneous because the difference equation (5) is time-invariant and noise process $w_{k}^{i}$ is i.i.d. for every $i=$ $\{1, \ldots, N\}$, and for any time-step $k$. Since the noise distribution is absolutely continuous with an everywherepositive density function, every subset of the state-space is accessible within one transition, i.e. the $d$-cycle is one, thus the Markov chain is aperiodic and $\psi$-irreducible, where $\psi$ is a non-trivial measure on the state space $\mathbb{R}^{n}$.

Remark 1 The probabilistic attribute of the policy (6) provides a design flexibility, via tuning the scheduling parameters, to achieve desired properties such as coping with data loss, and distributed implementation $[13,14]$.

\subsection{Preliminaries}

We employ the notion of $f$-ergodicity as the stability concept. A stochastic process is called ergodic if the timeaverage of its events over a sample sequence of transitions represents its overall behavior in the entire state-space.

Definition 1 [15, Ch. 10] Let the Markov chain $\Phi=$ $\left(\Phi_{0}, \Phi_{1}, \ldots\right)$ evolve in state space $X$, which is equipped with some known $\sigma$-algebra $\mathcal{B}(X)$. The Markov chain $\Phi$ is said to be positive Harris recurrent (PHR) if 
(1) a non-trivial measure $\nu(B)>0$ exists for a set $B \in \mathcal{B}$ such that for all $\Phi_{0} \in X, P\left(\Phi_{k} \in B, k<\infty\right)=1$ holds.

(2) $\Phi$ admits a unique invariant probability measure.

Definition $2[15$, Ch. 14] Let $f \geq 1$ be a real valued function in $\mathbb{R}^{n}$. A Markov chain $\Phi$ is said to be $f$-ergodic, if

(1) $\Phi$ is PHR with unique invariant measure $\pi$,

(2) the expectation $\pi(f) \triangleq \int f\left(\Phi_{k}\right) \pi\left(d \Phi_{k}\right)$ is finite,

(3) $\lim _{k \rightarrow \infty}\left\|P^{k}\left(\Phi_{0}, .\right)-\pi\right\|_{f}=0$ for every initial value $\Phi_{0} \in X$, where $\|\nu\|_{f} \triangleq \sup _{|g| \leq f}|\nu(g)|$.

Definition 3 Let $V: \mathbb{R}^{n} \rightarrow \mathbb{R}_{\geq 0}$, and $\Phi$ be a Markov chain. For any measurable function $V$, the drift $\Delta V(\cdot)$ is

$$
\Delta V\left(\Phi_{k}\right)=\mathrm{E}\left[V\left(\Phi_{k+1}\right) \mid \Phi_{k}\right]-V\left(\Phi_{k}\right), \quad \Phi_{k} \in \mathbb{R}^{n} .
$$

Theorem 1 (f-Norm Ergodic Theorem) [15, Ch. 14] Suppose that the Markov chain $\Phi$ is $\psi$-irreducible and aperiodic and let $f(\Phi) \geq 1$ be a real-valued function in $\mathbb{R}^{n}$. If a small set $\mathcal{D}$ and a non-negative real-valued function $V$ exist s.t. $\Delta V(\Phi) \leq-f(\Phi)$, for every $\Phi \in \mathbb{R}^{n} \backslash \mathcal{D}$, and $\Delta V<\infty$, for $\Phi \in \mathcal{D}$, the Markov chain $\Phi$ is $f$-ergodic.

Remark 2 [15, Ch. 5] All compact subsets of linear state-spaces are small sets and a small set is also petite.

In summary, an $f$-ergodic process converges to an invariant finite-variance measure over the entire state-space, confirming the Markov chain is a stationary process.

\section{Stochastic Stability}

In this section, stochastic stability of the described multiple-loop NCS is investigated in terms of $f$ ergodicity of the error Markov chain $e_{k}$. First, we select a non-negative quadratic function $V: \mathbb{R}^{n} \rightarrow \mathbb{R}_{\geq 0}$ as:

$$
V\left(e_{k}\right)=\sum_{i=1}^{N} e_{k}^{i^{\top}} Q^{i} e_{k}^{i}
$$

Due to the characteristics of the selected function $V$ in (10), $f$-ergodicity of the Markov chain (8) cannot always be guaranteed employing the drift $\Delta V$ over one transition step, i.e., for $k \rightarrow k+1$ as in (9). We illustrate this observation by constructing the following example. Illustrative example Let an NCS be composed of two identical scalar sub-systems competing for one channel slot. Assume $Q^{1}=Q^{2}=1$ and $e_{k}^{1}=e_{k}^{2}=\bar{e}_{k}>\lambda_{1}=\lambda_{2}$. The transmission chance for each sub-system is clearly $\frac{1}{2}$ according to (6). Employing (10), it is straightforward to show that the drift in (9), with $e_{k}=\left[e_{k}^{1} e_{k}^{2}\right]^{\top}$, becomes

$$
\Delta V\left(e_{k}\right)=\mathrm{E}\left[V\left(e_{k+1}\right) \mid e_{k}\right]-V\left(e_{k}\right)=2+\left\|A \bar{e}_{k}\right\|_{2}^{2}-2\left\|\bar{e}_{k}\right\|_{2}^{2} .
$$

For $A>\sqrt{2}$, the drift is positive, which violates the drift condition in Theorem 1. We show later that the ergodicity of Markov chain (8) is recovered considering the drift over an interval with multiple time-steps. Intuitively, only after all sub-systems have a chance to transmit, a negative drift $\Delta V$ over some interval of interest can be guaranteed. To fulfill this, we investigate the ergodicity of the Markov chain over the interval with length $N$. It should be mentioned that ergodicity over an interval implies ergodicity over any longer interval [15, Ch.19]. To infer $f$-ergodicity over an interval with length $N$, i.e. $[k, k+N]$, we modify the drift definition in (9) as follows:

$$
\Delta V\left(e_{k}, N\right)=\mathrm{E}\left[V\left(e_{k+N}\right) \mid e_{k}\right]-V\left(e_{k}\right), \quad e_{k} \in \mathbb{R}^{n} .
$$

Note that $e_{k+N}^{i}$ can be expressed as function of a previous state $e_{k+r_{i}}^{i}$ at a time-step $k+r_{i}$, with $r_{i} \in[0, N-1]$ :

$$
\begin{aligned}
e_{k+N}^{i} & =\prod_{j=r_{i}+1}^{N}\left(1-\delta_{k+j}^{i}\right) A_{i}^{N-r_{i}} e_{k+r_{i}}^{i} \\
& +\sum_{r=r_{i}}^{N-1}\left[\prod_{j=r+2}^{N}\left(1-\delta_{k+j}^{i}\right) A_{i}^{N-r-1} w_{k+r}^{i}\right]
\end{aligned}
$$

Theorem 2 Consider an NCS consisting of N heterogeneous LTI stochastic sub-systems modeled as (1), and a transmission channel subject to the constraint (7), and the control, estimation and scheduling laws given by (2), (3) and (6), respectively. Then for any $\lambda_{i} \in \mathbb{R}_{\geq 0}$ and positive definite $Q^{i}$, the Markov chain (8) is $f$-ergodic.

Proof To study stability, we let the NCS operate freely over the time interval $[k, k+N]$ under the scheduling policy (6), considering all possible outcomes in the statespace $\mathbb{R}^{n}$. To that end, we define at every $k^{\prime} \in[k, k+N]$ two time-varying, complementary and disjoint sets $S_{k^{\prime}}^{1}$ and $S_{k^{\prime}}^{2}$ such that for every $i \in\{1, \ldots, N\}, i \in S_{k^{\prime}}^{1}$ if $\left\|e_{k^{\prime}}^{i}\right\|_{Q^{i}}^{2} \leq \lambda_{i}$ and $i \in S_{k^{\prime}}^{2}$ if $\left\|e_{k^{\prime}}^{i}\right\|_{Q^{i}}^{2}>\lambda_{i}$. Note that, inclusion in either sets $S_{k^{\prime}}^{1}$ or $S_{k^{\prime}}^{2}$ depends not only on the transmission occasions but also on the random noise process. To take this into account, we discern the following three complementary and disjoint cases characterizing dynamics evolution of a sub-system $i$ in the state-space $\mathbb{R}^{n}$, over the time interval $[k, k+N]$. So, a sub-system $i$ :

$c_{1}$ : has either transmitted or not over $[k, k+N]$, but has not been eligible for transmission at $k+N$, i.e. $i \in S_{k+N-1}^{1}$, which implies $\left\|e_{k+N-1}^{i}\right\|_{Q^{i}}^{2} \leq \lambda_{i}$.

$c_{2}$ : has transmitted at least once over $[k, k+N]$, and has been eligible for transmission at $k+N$, i.e. $i \in$ $S_{k+N-1}^{2}$, which implies $\left\|e_{k+N-1}^{i}\right\|_{Q^{i}}^{2}>\lambda_{i}$.

$c_{3}$ : has not transmitted over $[k, k+N]$, i.e. $\delta_{k^{\prime}}^{i}=0$ for all $k^{\prime} \in[k, k+N]$, and has been eligible for transmission at $k+N$, i.e. $i \in S_{k+N-1}^{2}$, and $\left\|e_{k+N-1}^{i}\right\|_{Q^{i}}^{2}>\lambda_{i}$.

We investigate (11) for all cases in order to invoke Theorem 1. For all sub-systems $i \in c_{1}$, we know $\delta_{k+N}^{i}=0$. Employing the Cauchy-Schwarz inequality, we obtain

$$
\sum_{i \in c_{1}} \mathrm{E}\left[\left\|e_{k+N}^{i}\right\|_{Q^{i}}^{2} \mid e_{k}\right] \leq \sum_{i \in c_{1}} \lambda_{i}\left\|A_{i}\right\|_{2}^{2}+\operatorname{tr}\left(Q^{i}\right)
$$


For a sub-system $i \in c_{2}$, assume a transmission occurred at time $k+r_{i}$, i.e. $\delta_{k+r_{i}}^{i}=1$, where $r_{i} \in[0, N]$. Having statistical independence between the noise sequence $w_{k+r}^{i}$ and error state $e_{k+r_{i}-1}^{i}$, we have from (12)

$$
\sum_{i \in c_{2}} \mathrm{E}\left[\left\|e_{k+N}^{i}\right\|_{Q^{i}}^{2} \mid e_{k}\right] \leq \sum_{i \in c_{2}} \sum_{r=r_{i}}^{N} \operatorname{tr}\left(Q^{i}\right)\left\|A_{i}^{N-r}\right\|_{2}^{2}
$$

To infer $f$-ergodicity, we split the sub-systems $i \in c_{3}$ into two complementary and disjoint sub-cases, as follows:

$l_{1}^{c_{3}}$ : Sub-system $i$ has not transmitted over the interval $[k, k+N]$, but $i \in S^{1}$ at least once over $[k, k+N-1]$, $l_{2}^{c_{3}}$ : Sub-system $i$ has not transmitted over the interval $[k, k+N]$, and $i \in S^{2}$ at all those $N$ time-steps.

Within sub-case $l_{1}^{c_{3}}$, suppose that $k+r_{i}$ is the last time for which $i \in S_{k+r_{i}}^{1}$, which implies $\left\|e_{k+r_{i}}^{i}\right\|_{Q^{i}}^{2} \leq \lambda_{i}$. Knowing that $\delta_{k^{\prime}}^{i}=0$ for all $i \in c_{3}$, at all $k^{\prime} \in[k, k+N]$, we reach

$$
\begin{aligned}
& \sum_{i \in l_{1}^{c_{3}}} \mathrm{E}\left[\left\|e_{k+N}^{i}\right\|_{Q^{i}}^{2} \mid e_{k}\right] \leq \sum_{i \in l_{1}^{c_{3}}} \lambda_{i}\left\|A_{i}^{N-r_{i}}\right\|_{2}^{2} \\
& +\sum_{i \in l_{1}^{c_{3}}} \sum_{r=r_{i}}^{N-1} \operatorname{tr}\left(Q^{i}\right)\left\|A_{i}^{N-r-1}\right\|_{2}^{2} .
\end{aligned}
$$

All sub-systems $i \in l_{2}^{c_{3}}$ are in $S_{k^{\prime}}^{2}$, at all $k^{\prime} \in[k, k+N]$, since $\left\|e_{k^{\prime}}^{i}\right\|_{Q_{i}}^{2}>\lambda_{i}$. From (12) with $r^{\prime}=0$, it follows

$$
\begin{aligned}
& \sum_{i \in l_{2}^{c_{3}}} \mathrm{E}\left[\left\|e_{k+N}^{i}\right\|_{Q^{i}}^{2} \mid e_{k}\right] \leq \sum_{i \in l_{2}^{c_{3}}}\left[\left\|A_{i}^{N}\right\|_{2}^{2}\left\|e_{k}^{i}\right\|_{Q^{i}}^{2}\right] \\
& +\sum_{i \in l_{2}^{c_{3}}}\left[\sum_{r=1}^{N} \operatorname{tr}\left(Q^{i}\right)\left\|A_{i}^{N-r}\right\|_{2}^{2}\right] .
\end{aligned}
$$

The upper bound (16) depends on the initial value via the first term. As all the cases cannot happen all together, we calculate the probability that a sub-system $i$ belongs to $l_{2}^{c_{3}}$, under the scheduling policy (6). If subsystem $i$ does not transmit during the entire interval $[k, k+N]$, then there exists another sub-system, say $q$, which is awarded the channel access more than once. It is clear that $q \in c_{2}$. Let $k+r_{q}$ is the most recent time at which sub-system $q$ has transmitted, i.e. $\delta_{k+r_{q}}^{q}=1$. The probability that $q$ re-transmits at $k+N$, in presence of a sub-system $i \in l_{2}^{c_{3}}$ can be computed as follows:

$$
\begin{aligned}
& \mathrm{P}\left[\delta_{k+N}^{q}=1 \mid \delta_{k+r_{q}}^{q}=1, \delta_{k^{\prime}}^{i}=0,\left\|e_{k^{\prime}}^{i}\right\|_{Q^{i}}^{2}>\lambda_{i}\right] \\
& =\mathrm{E}\left[\mathrm{P}\left[\delta_{k+N}^{q}=1 \mid e_{k}\right] \mid \delta_{k+r_{q}}^{q}=1, \delta_{k^{\prime}}^{i}=0,\left\|e_{k^{\prime}}^{i}\right\|_{Q^{i}}^{2}>\lambda_{i}\right] \\
& =\mathrm{E}\left[\frac{\left\|e_{k+N-1}^{q}\right\|_{Q^{q}}^{2}}{\sum_{i \in c_{2}}\left\|e_{k+N-1}^{i}\right\|_{Q^{i}}^{2}+\sum_{i \in c_{3}}\left\|e_{k+N-1}^{i}\right\|_{Q^{i}}^{2}} \mid z_{i, q}\right] \\
& \leq \mathrm{E}\left[\frac{\left\|\sum_{r=r_{q}}^{N} A_{q}^{N-r} w_{k+r-2}^{q}\right\|_{Q^{q}}^{2}}{\sum_{i \in c_{2}} \lambda_{i}+\sum_{i \in l_{1}^{c_{3}}} \lambda_{i}+\sum_{i \in l_{2}^{c_{3}}}\left\|e_{k}^{i}\right\|_{Q^{i}}^{2}} \mid z_{i, q}\right] \\
& \leq \frac{\sum_{r=r_{q}}^{N} \operatorname{tr}\left(Q^{q}\right)\left\|A_{q}^{N-r}\right\|_{2}^{2}}{\sum_{i \in c_{2}} \lambda_{i}+\sum_{i \in l_{1}^{c_{3}}} \lambda_{i}+\sum_{i \in l_{2}^{c_{3}}}\left\|e_{k}^{i}\right\|_{Q^{i}}^{2}}=\mathrm{P}_{l_{2}^{c_{3}}},
\end{aligned}
$$

where $z_{i, q}$ abbreviates the conditions of the expectation, and the worst case scenario is considered which entails $\left\|e_{k^{\prime}}^{i}\right\|_{Q^{i}}^{2} \leq\left\|e_{k^{\prime}+1}^{i}\right\|_{Q^{i}}^{2}$, for all $i \in l_{2}^{c_{3}}$. From (17), probability of subsequent transmission for a sub-system, in presence of the ones with larger errors and no prior transmission, can be made arbitrarily close to zero by tuning $\lambda_{i}$ 's and $Q^{i}$ 's. Incorporating the occurrence probability for the case $l_{2}^{c_{3}}$, the $N$-step drift can be upper-bounded as

$$
\begin{aligned}
& \Delta V\left(e_{k}, N\right) \leq \sum_{i \in c_{1}, c_{2}, l_{1}^{c_{3}}} \mathrm{E}\left[\left\|e_{k+N}^{i}\right\|_{Q^{i}}^{2} \mid e_{k}\right] \\
& +\mathrm{P}_{l_{2}^{c_{3}}} \sum_{i \in l_{2}^{c_{3}}} \mathrm{E}\left[\left\|e_{k+N}^{i}\right\|_{Q^{i}}^{2} \mid e_{k}\right]-V\left(e_{k}\right) .
\end{aligned}
$$

Applying the probability (17) for the sub-case $l_{2}^{c_{3}}$, we find the upper bound for the $N$-step drift (18) as follows:

$$
\begin{aligned}
& \Delta V\left(e_{k}, N\right) \leq \sum_{i \in c_{1}} \lambda_{i}\left\|A_{i}\right\|_{2}^{2}+\operatorname{tr}\left(Q^{i}\right)+\sum_{i \in c_{2}} \sum_{r=r_{i}}^{N} \operatorname{tr}\left(Q^{i}\right)\left\|A_{i}^{N-r}\right\|_{2}^{2} \\
& +\sum_{i \in l_{1}^{c_{3}}}\left[\lambda_{i}\left\|A_{i}^{N-r_{i}}\right\|_{2}^{2}+\sum_{r=r_{i}}^{N-1} \operatorname{tr}\left(Q^{i}\right)\left\|A_{i}^{N-r-1}\right\|_{2}^{2}\right] \\
& +\mathrm{P}_{l_{2}^{c_{3}}} \sum_{i \in l_{2}^{c_{3}}}\left[\sum_{r=1}^{N} \operatorname{tr}\left(Q^{i}\right)\left\|A_{i}^{N-r}\right\|_{2}^{2}+\left\|A_{i}^{N}\right\|_{2}^{2}\left\|e_{k}^{i}\right\|_{Q^{i}}^{2}\right]-V\left(e_{k}\right) \\
& \leq \zeta_{b}^{+}+\frac{\sum_{r=r_{q}}^{N} \operatorname{tr}\left(Q^{q}\right)\left\|A_{q}^{N-r}\right\|_{2}^{2}}{\sum_{i \in c_{2}} \lambda_{i}+\sum_{i \in l_{1}^{c_{3}} \lambda_{i}}} \sum_{i \in l_{2}^{c_{3}}}\left[\sum_{r=1}^{N} \operatorname{tr}\left(Q^{i}\right)\left\|A_{i}^{N-r}\right\|_{2}^{2}\right] \\
& +\sum_{r=r_{q}}^{N} \operatorname{tr}\left(Q^{q}\right)\left\|A_{q}^{N-r}\right\|_{2}^{2} \sum_{i \in l_{2}^{c_{3}}}\left\|A_{i}^{N}\right\|_{2}^{2}-V\left(e_{k}\right), \quad \text { (19) }
\end{aligned}
$$

with $\zeta_{b}^{+}$representing all the bounded positive terms correspond to the cases $c_{1}, c_{2}, l_{1}^{c_{3}}$, and (19) is ensured since $\left\|e_{k}^{i}\right\|_{Q^{i}}^{2} \leq \sum_{j=1}^{N}\left\|e_{k}^{j}\right\|_{Q^{j}}^{2}=V\left(e_{k}\right)$, for every $i \in$ $\{1, \ldots, N\}$. Define $f\left(e_{k}\right)=\epsilon V\left(e_{k}\right)-\zeta_{b}^{+}-\xi_{b}^{+}$, where $\epsilon \in(0,1]$, and $\xi_{b}^{+}$stands for the second and third terms in the right side of the latest inequality. Then, we can find a small set $\mathcal{D}$ and an $\epsilon$ such that $f\left(e_{k}\right) \geq 1$, and $\Delta V\left(e_{k}, N\right) \leq-f$, which readily proves $f$-ergodicity of the Markov chain (8) according to the Theorem 1.

Remark 3 Theorem 2 ensures the Markov chain (8) visits compact sets $\mathcal{D} \subset \mathbb{R}^{n}$ at most every $N$ time-steps. The boundary of $\mathcal{D}$, however, varies with parameters $\lambda_{i}$, $Q^{i}, N$, and $A_{i}$, as it is discussed in Section 4 .

Corollary 1 The multiple-loop NCS described in (1)(5) with the overall network state $\left[x_{k}^{T}, e_{k}^{T}\right]^{T}$, under the scheduling law (6) is Lyapunov mean square stable.

Proof An LTI system with state vector $X_{k}$ is said to be Lyapunov mean square stable (LMSS) if given $\varepsilon>0$, there exists $\rho(\varepsilon)$ s. t. $\left\|X_{0}\right\|_{2}<\rho$ implies $\sup _{k \geq 0} \mathrm{E}\left[\left\|X_{k}\right\|_{2}^{2}\right] \leq \varepsilon,[10]$. Let $X_{k}=\left[x_{k}^{\top} e_{k}^{\top}\right]^{\top}$. The overall NCS state is LMSS if $\sum_{c_{j}} \mathrm{P}_{c_{j}} \mathrm{E}\left[\left\|e_{k}^{i}\right\|_{2}^{2} \mid i \in c_{j}\right] \leq \varepsilon$, assuming that the stabilizing control gains $L_{i}$ exist for 
all $i \in\{1, \ldots, N\}$. Starting from time $k$, uniform upperbounds for $\mathrm{E}\left[\left\|e_{k+N}^{i}\right\|_{2}^{2}\right]$ for sub-systems $i \in\left\{c_{1}, c_{2}, l_{1}^{c_{3}}\right\}$ are derived in (13)-(15) over $N$-step intervals, setting $\mathrm{P}_{c_{j}}=1$. For the case $l_{2}^{c_{3}}$, the uniform upper-bound for $\mathrm{P}_{l_{2}^{c_{3}}} \mathrm{E}\left[\left\|e_{k}^{i}\right\|_{2}^{2} \mid i \in l_{2}^{c_{3}}\right]$ is obtained in (19), excluding $\zeta_{b}^{+}-V\left(e_{k}\right)$. Note that LMSS implies $f$-ergodicity but not vice-versa since the latter holds not only with quadratic Lyapunov function but also with $p$-power variations.

\section{Performance Bounds}

To investigate performance of the scheduler (6), we define the following per-time-step cost function for all $i \in$ $\{1, \ldots, N\}$, associated with weight matrices $Q^{i}>0$ :

$$
J_{e_{k}}=\sum_{j=1}^{N} e_{k}^{j^{\top}} Q^{j} e_{k}^{j}+\eta \delta_{k}^{j}=\sum_{j=1}^{N}\left\|e_{k}^{j}\right\|_{Q^{j}}^{2}+\eta \delta_{k}^{j},
$$

where $\eta \geq 0$ denotes the cost of channel utilization.

Remark 4 According to (20), we solely evaluate the performance of the scheduler, i.e. there is no penalty on the signals $u_{k}^{i}$ and $x_{k}^{i}$. Due to the independence of control inputs from the scheduling policy, terms involving $u_{k}^{i}$ and $x_{k}^{i}$ can be readily added to the cost function. A potential minimization of those terms directly affects the design of gain $L_{i}$, which is out of scope of this work.

Lemma 1 [5] Let $e_{k}$ be a Markov chain with state space $X$. Introduce $J_{e_{k}}: X \rightarrow \mathbb{R}$ and a measurable function $h: X \rightarrow \mathbb{R}$, where $h\left(e_{k}\right) \geq 0$ for all $e_{k} \in X$. Define the average cost $J_{\text {ave }}=\lim _{T \rightarrow \infty} \sup \frac{1}{T} \sum_{k=0}^{T-1} \mathrm{E}\left[J_{e_{k}}\right]$. Then

$$
J_{\text {ave }} \leq \sup _{e_{k} \in X}\left\{J_{e_{k}}+\mathrm{E}\left[h\left(e_{k+1}\right) \mid e_{k}\right]-h\left(e_{k}\right)\right\} .
$$

Lemma 1 provides the upper-bound according to one step time-transition of $h$ function. Since $e_{k}$ is $\psi$ irreducible and evolves in the uncountable space $\mathbb{R}^{n}$, one can generate a Markov chain by sampling the states of the original chain at steps $\{0, N, 2 N, \ldots\}$. It is straightforward to show that the generated Markov chain is also $\psi$-irreducible, aperiodic, and time-homogeneous [15, Ch. 1]. This facilitates the upper-bound on the average cost $J_{\text {ave }}$ to be defined over an $N$ time-step interval as follows

$$
J_{\text {ave }} \leq \sup _{e_{k} \in X}\left\{J_{e_{k}}+\mathrm{E}\left[h\left(e_{k+N}\right) \mid e_{k}\right]-h\left(e_{k}\right)\right\}
$$

Let $h\left(e_{k}\right)=\sum_{i=1}^{N}\left\|e_{k}^{i}\right\|_{Q^{i}}^{2}$, then from (20), we conclude

$$
J_{\text {ave }} \leq \sup _{e_{k} \in X} \sum_{i=1}^{N}\left[\mathrm{E}\left[\left\|e_{k+N}^{i}\right\|_{Q^{i}}^{2} \mid e_{k}\right]+\eta \delta_{k}^{i}\right]
$$

Theorem 3 Consider the NCS described in Theorem 2. Then the average cost $J_{\text {ave }}=\lim _{T \rightarrow \infty} \sup \frac{1}{T} \sum_{k=0}^{T-1} \mathrm{E}\left[J_{e_{k}}\right]$ is upper-bounded as a strictly increasing function of $\left\|A_{i}\right\|, \operatorname{tr}\left(Q^{i}\right), N$, and $\eta$, over all initial conditions and for all $i \in\{1, \ldots, N\}$, while the dependency on $\lambda_{i}$ 's is convex.
Proof We derive uniform upper-bounds for the average cost (22) for each case $c_{1}, c_{2}$ and $c_{3}$, considering the NCS model described in Theorem 2. For the cases $c_{1}, c_{2}$, and $l_{1}^{c_{3}}$ the upper-bounds derived in (13)-(15) are valid and only the communication cost should be added. Therefore, we derive the followings for the average cost (22):

$$
\begin{aligned}
J_{\text {ave }}^{i \in c_{1}} & \leq \sum_{c_{1}} \lambda_{i}\left\|A_{i}\right\|_{2}^{2}+N \eta+\operatorname{tr}\left(Q^{i}\right), \\
J_{\text {ave }}^{i \in c_{2}} & \leq N \eta+\sum_{c_{2}} \sum_{r=r_{i}}^{N} \operatorname{tr}\left(Q^{i}\right)\left\|A_{i}^{N-r}\right\|_{2}^{2}, \\
J_{\text {ave }}^{i \in l_{1}^{c_{3}}} & \leq \sum_{l_{1}^{c_{3}}}\left[\lambda_{i}\left\|A_{i}^{N-r_{i}}\right\|_{2}^{2}+\sum_{r=r_{i}}^{N-1}\left\|A_{i}^{N-r-1}\right\|_{2}^{2} \operatorname{tr}\left(Q^{i}\right)\right],
\end{aligned}
$$

where, $N \eta$ upper-bounds the cost of communication for cases $c_{1}$ and $c_{2}$. Note that no transmission occurs within case $c_{3}$. For sub-systems in $l_{2}^{c_{3}}$, the non-uniform upperbound (16) is valid. Therefore, according to (22), we have

$J_{\text {ave }}^{i \in l_{2}^{c_{3}}} \leq \sup _{e_{k}} \sum_{l_{2}^{c_{3}}}\left\|A_{i}^{N}\right\|_{2}^{2}\left\|e_{k}^{i}\right\|_{Q^{i}}^{2}+\sum_{r=1}^{N} \operatorname{tr}\left(Q^{i}\right)\left\|A_{i}^{N-r}\right\|_{2}^{2}$.

We rewrite the average cost (22) for different cases as $J_{\text {ave }}=\mathrm{P}_{c_{1}} J_{\text {ave }}^{i \in c_{1}}+\mathrm{P}_{c_{2}} J_{\text {ave }}^{i \in c_{2}}+\mathrm{P}_{l_{1}^{c_{3}}} J_{\text {ave }}^{i \in l_{1}^{c_{3}}}+\mathrm{P}_{l_{2}^{c_{3}}} J_{\text {ave }}^{i \in l_{2}^{c_{3}}}$. Since $\delta_{\bar{k}}=0$ for all sub-systems $i \in l_{2}^{c_{3}}$ and for all $\bar{k} \in[k+N]$, $J_{\text {ave }}^{i \in l_{2}^{c_{3}}} \leq \sup _{e_{k} \in \mathbb{R}^{n}} \sum_{i \in l_{2}^{c_{3}}} \mathrm{E}\left[\left\|e_{k+N}^{i}\right\|_{Q^{i}}^{2} \mid e_{k}\right]$. Given the probability of occurrence for the sub-case $l_{2}^{c_{3}}$ in (17), and considering the worst-case scenario, we conclude

$$
\begin{aligned}
\mathrm{P}_{l_{2}^{c_{3}}} J_{\text {ave }}^{i \in l_{2}^{c_{3}}} \leq & \frac{\sum_{r=r_{q}}^{N} \operatorname{tr}\left(Q^{q}\right)\left\|A_{q}^{N-r}\right\|_{2}^{2}}{\sum_{i \in c_{2}} \lambda_{i}+\sum_{i \in l_{1}^{c_{3}} \lambda_{i}}} \sum_{i \in l_{2}^{c_{3}}} \sum_{r=1}^{N} \operatorname{tr}\left(Q^{i}\right)\left\|A_{i}^{N-r}\right\|_{2}^{2} \\
& +\sum_{r=r_{q}}^{N} \operatorname{tr}\left(Q^{q}\right)\left\|A_{q}^{N-r}\right\|_{2}^{2} \sum_{i \in l_{2}^{c_{3}}}\left\|A_{i}^{N}\right\|_{2}^{2} .
\end{aligned}
$$

Summing up the uniform upper-bounds (23)-(26) for all cases $\left\{c_{1}, c_{2}, c_{3}\right\}$, we obtain overall upper-bound for the average cost function (22) independent of the initial state. The overall upper-bound is strictly increasing w.r.t. $\left\|A_{i}\right\|, N, \operatorname{tr}\left(Q^{i}\right)$, and $\eta$, for all $i \in\{1, \ldots, N\}$. Since, the error thresholds $\lambda_{i}$ 's are determined locally for each sub-system $i$, and moreover the obtained overall upper-bound for the average cost is the summation of local functions which are convex w.r.t. their corresponding local threshold $\lambda_{i}$, the overall upper-bound is convex w.r.t. the $\lambda_{i}$ 's, and the proof then readily follows.

Intuitively, lowering $\lambda_{i}$ 's implies that more sub-systems compete for channel access which increases the probability that sub-systems with lower priorities transmit and consequently increases the average cost. On the other hand, setting $\lambda_{i}$ 's higher results in more sub-systems being deterministically kept out of channel access competition and subsequently they remain in open-loop. This convex property in the error thresholds enables us to search for the unique minimizing $\lambda_{i}$ 's, resulting in the sub-optimal upper-bound for the average cost (22). 


\section{$5 \quad$ Numerical Results}

We consider NCSs comprised of two heterogeneous system classes each consisting of homogeneous scalar subsystems. The first class includes $\frac{N}{2}$ control loops with unstable plants and parameters $A_{1}=1.25, B_{1}=1$, while the second class contains $\frac{N}{2}$ loops with stable processes with parameters $A_{2}=0.75$, and $B_{2}=1$. We select $x_{0}^{i}=0$ for each sub-system $i$, and $w_{k}^{i} \sim \mathcal{N}(0,1)$. To control the plants, deadbeat control laws $L_{i}=A_{i}$, and model-based estimator (3) are used. For simplicity, we select $Q^{i}=I$. Fig. 2 shows the changes in error variance (8) w.r.t. the local thresholds $\lambda$, for NCSs with different number of sub-systems, under the scheduling policy (6). It suggests that it is possible to tune the thresholds optimally in order to minimize the error variance. Expectedly, the optimal value of $\lambda$ monotonically increases by increasing $N$, because the competition among sub-systems to access the sole channel slot intensifies with increasing $N$.

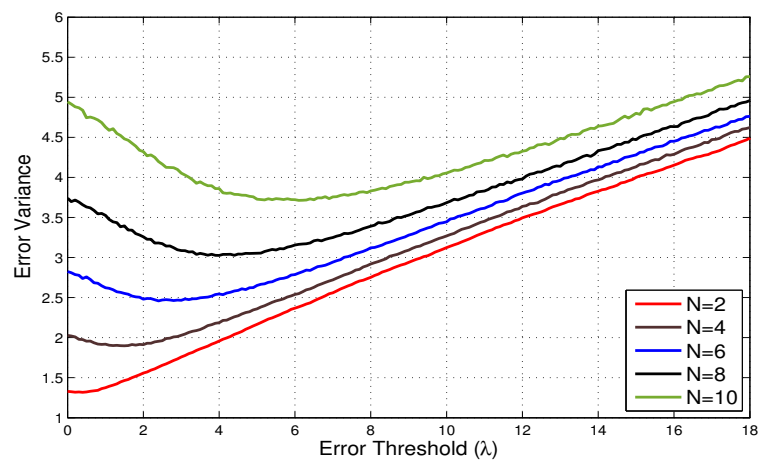

Fig. 2. Error variance vs. local error thresholds.

Fig. 3 provides comparisons in terms of average error variance between our bi-character policy, TOD, TDMA, CSMA, event-triggered threshold policy [16], and event-triggered pure probabilistic scheme [12], for different NCS setups with $N \in\{2,4,6,8,10,20\}$, subject to the constraint (7). Note that for $N>2$, we have more unstable sub-systems than the transmission slots per time-step. The variances are calculated via Monte Carlo simulation with $2 \times 10^{5}$ samples. For the results to be comparable, we disregard the communication penalty, i.e. $\eta=0$. We calculate the variances setting equal $\lambda$ 's for all $N$ sub-systems in a certain NCS setup. The chosen thresholds are the optimal values according to Fig. 2.

For fair comparisons, we derived the optimal TDMA pattern by brute force search over a finite time window (infinite horizon optimal TDMA pattern is NP-hard). The optimal search is however extremely exhaustive, while the pattern changes sensitively with the changes of system parameters. For the CSMA scheme, the transmission probability for a sub-system $i$ is pre-given by $\frac{A_{i}^{2}}{\sum_{j=1}^{N} A_{j}^{2}}$ at each time-step. As seen in Fig. 3, this protocol results in an acceptable performance up to $N=4$. This is expected as the static CSMA may result in a

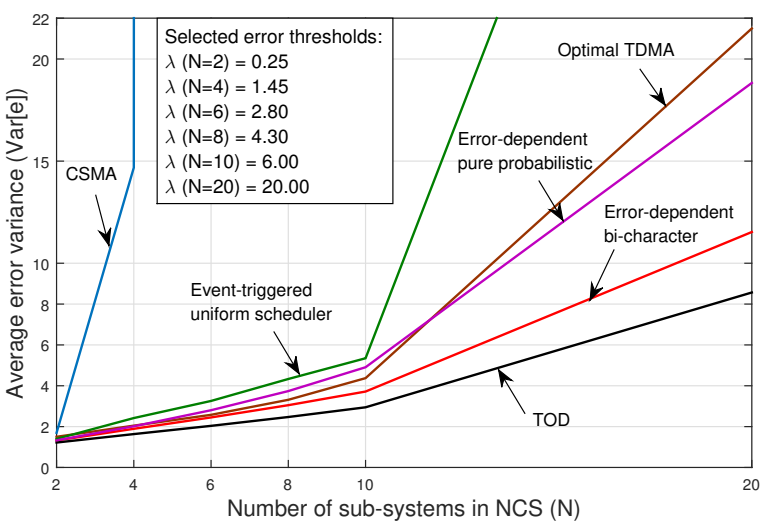

Fig. 3. Comparison of the average error variance.

lengthy non-transmission period for unstable systems. To highlight the convenience of deterministic feature of the policy (6), the pure probabilistic approach, which considers all sub-systems for channel access competition at every time-step, i.e. $\lambda_{i}=0$, for all $i$, is additionally investigated. Expectedly, the error variance grows compared to the bi-character scheme, as sub-systems with small errors might use the channel in the presence of those with larger errors. A bi-character scheme proposed in [16] which disregards the error-dependent prioritization in policy (6) and instead employs a randomization with uniform probabilities to selects $c$ sub-systems for transmission, in case $j_{\lambda}>c$. Fig. 3 shows that our approach outperforms all mentioned schemes, especially when $N$ increases. Moreover, the performance of the bi-character approach closely follows the TOD scheme. Table 1 provides analytic upper-bounds for the average cost (22), according to the expressions (23)-(26), for NCSs with $N=\{2,4,6,8,10\}$. The upper-bounds are calculated for the analytic optimal error thresholds derived from (23)-(26), and they do not necessarily equal the optimal thresholds obtained from simulation. For simplicity, we assume $\eta_{i}=\lambda_{i}$. Recall that the upper bounds are derived for the worst case scenario and they are conservative compared to the simulation results.

Table 1

Analytic upper bounds for the average cost (22).

\begin{tabular}{l|ccccc} 
Number of loops $(N)$ & 2 & 4 & 6 & 8 & 10 \\
\hline Error threshold $(\lambda)$ & 0.28 & 6 & 10 & 15 & 18 \\
\hline Upper bound on $J_{\text {ave }}$ & 3.12 & 3.99 & 6.14 & 12.28 & 25.60
\end{tabular}

\section{Conclusion}

We present an error-dependent scheduling policy for multi-loop shared-resource NCSs. The scheduler is capable of allocating communication resources more efficiently based on an error-dependent priority measure. Given stabilizing feedback controllers, we show convergence of the network-induced error, and therefore 
stability of the overall multi-loop NCS. We furthermore derive analytical performance bounds for an average cost function. Simulation results illustrate major performance improvements in terms of the overall error variance in comparison with the conventional schemes.

\section{References}

[1] K.J. Åström and B.M. Bernhardsson. Comparison of Riemann and Lebesgue sampling for first order stochastic systems. In 41st IEEE Conf. on Decision and Control, pages 2011-2016 vol.2, 2002.

[2] R. Blind and F. Allgöwer. Analysis of Networked EventBased Control with a Shared Communication Medium: Part II - Slotted ALOHA. In 18th IFAC World Congress, pages $8830-8835,2011$

[3] A. Cervin and T. Henningsson. Scheduling of event-triggered controllers on a shared network. In 47 th IEEE Conference on Decision and Control, pages 3601-3606, 2008.

[4] D. Christmann, R. Gotzhein, S. Siegmund, and F. Wirth. Realization of TOD in wireless multihop networks. IEEE Trans. on Industrial Informatics, 10(1):17-26, 2014.

[5] R. Cogill, S. Lall, and J.P. Hespanha. A constant factor approximation algorithm for event-based sampling. In American Control Conference, pages 305-311, 2007.

[6] D.V. Dimarogonas and K.H. Johansson. Event-triggered control for multi-agent systems. In 48th IEEE Conf. on Decision and Control held jointly with 28th Chinese Control Conf., pages 7131-7136, 2009.

[7] M.C.F. Donkers, W.P.M.H. Heemels, D. Bernardini, A. Bemporad, and V. Shneer. Stability analysis of stochastic networked control systems. Automatica, 48(5):917-925, 2012.

[8] C. Feng, R. German, and F. Dressler. Towards IEEE 802.15.4e: A study of performance aspects. In IEEE 8th International Conf. on Pervasive Computing and Communications Workshops, pages 68-73, 2010.

[9] J.P. Hespanha, P. Naghshtabrizi, and Yonggang Xu. A survey of recent results in networked control systems. Proceedings of the IEEE, 95(1):138-162, 2007.

[10] F. Kozin. A survey of stability of stochastic systems. Automatica, 5(1):95-112, 1969.

[11] J. Lunze and D. Lehmann. A state-feedback approach to event-based control. Automatica, 46(1):211-215, 2010.

[12] M.H. Mamduhi, A. Molin, and S. Hirche. Event-based scheduling of multi-loop stochastic systems over shared communication channels. In 21st Int. Symp. on Mathematical Theory of Networks and Systems, pages 266-273, 2014.

[13] M.H. Mamduhi, D. Tolic, and S. Hirche. Decentralized eventbased scheduling for shared-resource networked control systems. In 14th European Control Conf., pages 941-947, 2015.

[14] M.H. Mamduhi, D. Tolic, and S. Hirche. Robust event-based data scheduling for resource constrained networked control systems. In American Control Conf., pages 4695-4701, 2015.

[15] S.P. Meyn and R.L. Tweedie. Markov chains and stochastic stability. Springer London, 1996.

[16] A. Molin and S. Hirche. A bi-level approach for the design of event-triggered control systems over a shared network. Discrete Event Dynamic Systems, 24(2):153-171, 2014.

[17] A. Molin and S. Hirche. Price-based adaptive scheduling in multi-loop control systems with resource constraints. IEEE Trans. on Automatic Control, 59(12):3282-3295, Dec 2014.
[18] D. Nesic and A.R. Teel. Input-output stability properties of networked control systems. IEEE Trans. on Automatic Control, 49(10):1650-1667, 2004.

[19] C. Ramesh, H. Sandberg, and K.H. Johansson. Stability analysis of multiple state-based schedulers with csma. In 51st IEEE Conf. on Decision and Control, pages 7205-7211, 2012.

[20] Y. Shunyuan, T. Korakis, and S. Panwar. CSMAC: A new centralized scheduling-based MAC protocol for wireless LAN. In IEEE Wireless Communications and Networking Conf., pages 1-6, 2009.

[21] M. Tabbara and D. Nesic. Input-output stability of networked control systems with stochastic protocols and channels. IEEE Trans. on Automatic Control, 53(5):11601175, 2008.

[22] P. Tabuada. Event-triggered real-time scheduling of stabilizing control tasks. IEEE Trans. on Automatic Control, 52(9):1680-1685, 2007.

[23] G. C. Walsh, H. Ye, and L. G. Bushnell. Stability analysis of networked control systems. IEEE Trans. on Control Systems Technology, 10(3):438-446, 2002.

[24] X. Wang and M.D. Lemmon. Event-triggering in distributed networked control systems. IEEE Trans. on Automatic Control, 56(3):586-601, 2011.

[25] S. Zhou and Z. Zhang. cMAC: A centralized MAC protocol for high speed wireless LANs. In IEEE Conf. on Global Telecommunications, pages 1-6, 2011. 